\title{
Whole-pattern profile fitting of powder diffraction data collected in parallel-beam flat-plate asymmetric reflection geometry
}

\author{
Matthew R. Rowles and Ian C. Madsen
}

J. Appl. Cryst. (2010). 43, 632-634

\section{IUCr Journals CRYSTALLOGRAPHY JOURNALS ONLINE}

Copyright (C) International Union of Crystallography

Author(s) of this paper may load this reprint on their own web site or institutional repository provided that this cover page is retained. Republication of this article or its storage in electronic databases other than as specified above is not permitted without prior permission in writing from the IUCr.

For further information see http://journals.iucr.org/services/authorrights.html 
Journal of

Applied

Crystallography

ISSN 0021-8898

Received 1 October 2009

Accepted 1 March 2010

(C) 2010 International Union of Crystallography Printed in Singapore - all rights reserved

\section{Whole-pattern profile fitting of powder diffraction data collected in parallel-beam flat-plate asymmetric reflection geometry}

\author{
Matthew R. Rowles ${ }^{a, b *}$ and Ian C. Madsen ${ }^{a}$ \\ ${ }^{\mathbf{a}} \mathrm{CSIRO}$ Process Science and Engineering, Box 312, Clayton South, Victoria 3168, Australia, and ${ }^{\mathbf{b}} \mathrm{CSIRO}$ Light \\ Metals Flagship, Box 312, Clayton South, Victoria 3168, Australia. Correspondence e-mail: \\ matthew.rowles@csiro.au
}

\begin{abstract}
A simple, physically based model that allows the whole-pattern profile fitting of diffraction data collected in parallel-beam flat-plate asymmetric reflection geometry is presented. In this arrangement, there is a fixed angle between the incident beam and the sample, resulting in a fixed-length beam footprint. The use of a wide-angle detector for the simultaneous detection of the data precludes the use of any diffracted beam optics. Therefore, the observed peak widths are a function of the length of the beam footprint on the sample. The model uses up to three refinable parameters, depending on the intensity profile of the beam, to calculate the effect of diffraction angle on the width of all diffracted peaks. The use of this model reduces the total number of parameters required to fit the observed peak widths and shapes, hence leading to increased stability in the profile analysis. Implementations of the model are provided for both fundamental parameters and empirical approaches.
\end{abstract}

\section{Introduction}

Flat-plate asymmetric reflection geometry is a useful arrangement for diffraction studies of surface phenomena and in situ experimentation, and is the only geometry possible when using a flat-plate sample with a wide-angle position-sensitive detector, such as the INEL CPS120 in laboratory instruments or the Mythen detector (Schmitt et al., 2003) as used at a number of synchrotron facilities. As the use of this geometry becomes more widespread, it becomes increasingly important to correctly model the effects of the instrument on the measured diffraction patterns. Toraya \& Yoshino (1994) have demonstrated the corrections for a pseudo-parallel beam using a modified Thompson-Cox-Hastings model (Thompson et al., 1987); however, the geometry of the instrument described by Toraya and Yoshino included a Ge(111) analyser crystal in the diffracted beam, which has a significant impact on the observed peak widths and shapes. In the configuration described here, there are no diffracted beam optics; therefore, it is the size and intensity distribution of the footprint of the incident beam on the sample that governs the observed peak widths and shapes, and hence the overall peak resolution. This paper describes the development of a fundamental parameters model which uses the known instrument settings to calculate the instrument component of width.

In this asymmetric geometry, the observed intensity has a different variation as a function of diffraction angle compared with conventional Bragg-Brentano arrangements (Toraya et al., 1993), and the effect of sample displacement is greatly enhanced (Masson et al., 1996). In addition to these effects, the width of the peak profiles changes significantly with increasing diffraction angle (see Fig. 1) owing to the $2 \theta$ dependence of the apparent size of the beam on the sample, which is due to the absence of any diffracted beam optics.

Assuming a parallel incident beam, the width, $w$, of the diffracted beam, in degrees, is given by

$$
w=\frac{b}{\sin (\alpha)} \frac{\sin (2 \theta-\alpha)}{R} \frac{180}{\pi},
$$

where $b$ is the beam height, $\alpha$ is the angle of the sample with respect to the incident beam (beam angle), $R$ is the radius of the detector and $2 \theta$ is the diffraction angle. The first term is the actual length of the beam on the sample (beam footprint) and the second term modifies the beam footprint to give the apparent length as seen at a given diffraction angle. The width of the diffracted beam goes through a maximum at an angle perpendicular to the sample surface. All

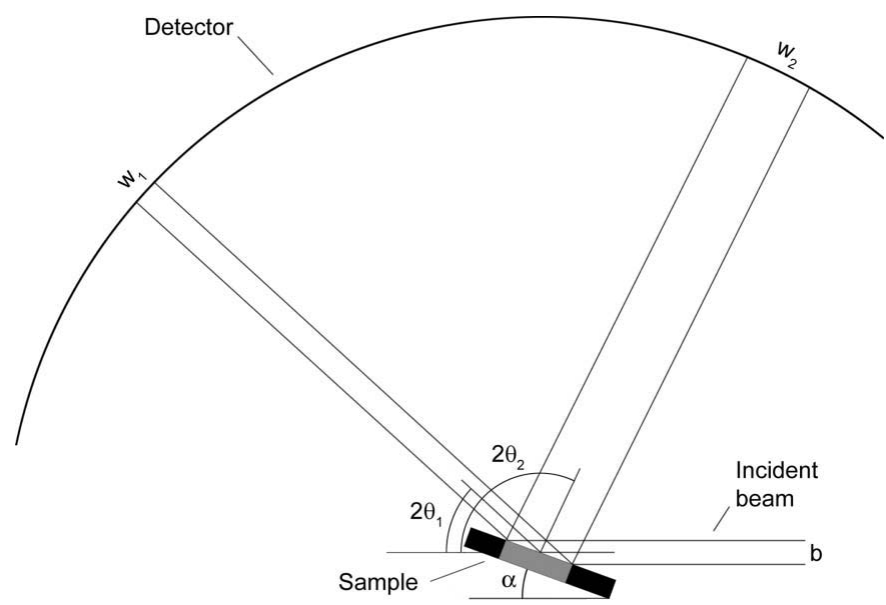

Figure 1

A schematic diagram showing the effect of diffraction angle $(2 \theta)$ on the width $(w)$ of the diffracted beam in flat-plate asymmetric reflection geometry. The height of the beam is given by $b$; the angle of the sample with respect to the incident beam (beam angle) is given by $\alpha$. The highlighted section of the sample indicates the beam footprint. 
Table 1

Parameters from the Rietveld refinement of yttrium oxide at various incident beam angles and heights using a fundamental parameters model and a TCHZ model.

Beam heights that were nominally equal were constrained to be equal. The mixing parameters ( $\eta=1$ is pure hat) for nominally equal beam heights were constrained to be equal.

\begin{tabular}{|c|c|c|c|c|c|c|}
\hline \multirow[b]{2}{*}{$\begin{array}{l}\text { Beam angle } \\
\left(^{\circ}\right)\end{array}$} & \multirow[b]{2}{*}{$\begin{array}{l}\text { Nominal } \\
\text { beam height } \\
(\mathrm{mm})\end{array}$} & \multicolumn{3}{|c|}{ Fundamental parameters model } & \multicolumn{2}{|l|}{ TCHZ model } \\
\hline & & $\begin{array}{l}\text { Refined } \\
\text { beam height } \\
(\mathrm{mm})\end{array}$ & $\begin{array}{l}\text { Mixing } \\
\text { parameter } \\
(\eta)\end{array}$ & $r_{\text {Bragg }}$ & $\begin{array}{l}\text { Refined } \\
\text { 'beam height' } \\
(\mathrm{mm})\end{array}$ & $r_{\text {Bragg }}$ \\
\hline 4.000 & 0.1 & 0.057 & 0.05 & 2.56 & 0.051 & 3.30 \\
\hline 8.011 & 0.1 & 0.057 & 0.05 & 4.04 & 0.051 & 4.16 \\
\hline 15.99 & 0.138 & 0.096 & 0.76 & 2.53 & 0.048 & 2.51 \\
\hline 4.000 & 0.5 & 0.30 & 0.75 & 2.71 & 0.17 & 3.13 \\
\hline 8.011 & 0.5 & 0.30 & 0.75 & 2.27 & 0.17 & 2.48 \\
\hline 15.99 & 0.5 & 0.30 & 0.75 & 2.03 & 0.17 & 1.85 \\
\hline 32.00 & 0.5 & 0.30 & 0.75 & 3.50 & 0.17 & 3.71 \\
\hline
\end{tabular}

parameters can be measured experimentally, allowing the values to be fixed, or at least constrained, when refining the model.

\section{Experimental}

\subsection{Model formulation}

Given an ideal parallel incident beam with a uniform intensity distribution across its height, and following a fundamental parameters approach to profile fitting (Alexander, 1954; Cheary \& Coelho, 1992), the most appropriate peak width convolution to include in a refinement is a hat function with its width given by equation (1). To allow for non-ideal beam intensity profiles, such as a drop off in the intensity towards the edges of the beam, the hat function can be replaced by another function, or additional convolutions and mixing parameters can be introduced: for example, to include a Gaussian component,

$$
w=\eta \times \text { hat }+(1-\eta) \times \text { Gaussian, }
$$

where the mixing parameter, $\eta$, is constrained to the range $0-1$. The fundamental parameters model used in the following analysis utilizes both hat and Gaussian convolutions with a single mixing parameter.

An empirical approach to fitting the profile shapes, such as the Thompson-Cox-Hastings model (Thompson et al., 1987; Young \& Desai, 1989), adequately models the profile shapes; however, the parameter values will have no physical correlation with crystallite size or microstrain. To allow for a more physically realistic model, and to allow direct comparison of data collected from the same sample with different beam heights and angles, or to allow the application of parameters derived from standards, the contribution to the peak width due to the beam footprint should be separated from the other instrumental and sample-related components. To this end, the halfwidth, in degrees, of the Gaussian component of the pseudo-Voigt peaks in the Thompson-Cox-Hastings model needs to be modified as follows:

$H_{\mathrm{G}}=\left(U \tan ^{2} \theta+V \tan \theta+W+\frac{Z}{\cos ^{2} \theta}\right)^{1 / 2}+\frac{b}{\sin (\alpha)} \frac{\sin (2 \theta-\alpha)}{R} \frac{180}{\pi}$,

where $U, V, W$ and $Z$ have their standard definitions (Thompson et al., 1987; Young \& Desai, 1989). In this approach, following the work of Toraya \& Yoshino (1994), the beam height, $b$, loses its association with the physically measurable beam height owing to the assumption of a totally Gaussian beam intensity profile - with a large beam height, the beam intensity profile approximates a hat ( $c f$. Table 1), which can be fitted with a Gaussian only to the detriment of other profile parameters. The final mix of Lorentzian and Gaussian components in the pseudo-Voigt peaks is then calculated as given by Thompson et al. (1987).

The application of either beam width correction, in conjunction with the sample displacement and transparency corrections of Masson et al. (1996) and the intensity correction of Toraya et al. (1993), changes the functional form of these factors coded into the majority of Rietveld analysis programs, which most often assume Bragg-Brentano or capillary geometry. These corrections, particularly using the fundamental parameters approach, allow for the refinement of physically realistic intensity, displacement and peak width models during analyses of data collected in non-standard geometries.

\subsection{Data collection}

Diffraction data were obtained from an yttrium oxide (cubic, $a=$ $10.6040 \AA$ ) sample in flat-plate asymmetric reflection geometry at the powder diffraction beamline (Wallwork et al., 2007) of the Australian Synchrotron with seven combinations of beam height and beam angle (see Table 1). The incident beam energy $(8.00131 \mathrm{keV})$ and $2 \theta$ zero error were determined from a standard $0.3 \mathrm{~mm}$ capillary of an $\mathrm{LaB}_{6} /$ diamond mixture. The beam height was varied by altering slits in the incident beam, whilst its width remained constant. The beam angle was varied by rotating the sample with the $\omega$ circle of the goniometer.

\section{Results}

Two models were constructed to allow the simultaneous refinement of all diffraction patterns using the Rietveld (1969) method as implemented in TOPAS (Version 4; Bruker, 2008) - the first followed a fundamental parameters approach, the other utilized the TCHZ profile model as described by Young \& Desai (1989) with the modified Gaussian half-width given in equation (3).

For both models, the $2 \theta$ zero error was fixed to the value determined from the standard measurement, and the beam angle was fixed to the physically measured value because of the strong correlation between beam height and angle in the refinement. The background and scale factors were refined independently for each pattern. The instrument configuration function, structure model (Ishibashi et al., 1994) and sample displacement correction (Masson et al., 1996) [see equation (4); $s$ is the displacement, $R$ is the detector radius] were refined with the constraint that they were the same across all patterns in both models:

$$
\Delta 2 \theta=-\frac{s \sin (2 \theta)}{R \sin (\alpha)} \frac{180}{\pi}
$$

Intensities were scaled according to the correction factor, $S_{\text {int }}$, given by Toraya et al. (1993):

$$
S_{\text {int }}=2 /\left[1+\frac{\sin (\alpha)}{\sin (2 \theta-\alpha)}\right] .
$$

In both models, the beam heights were refined but constrained to be the same for those patterns collected with equal incident beam slit settings. Sample transparency (after Masson et al., 1996) was allowed for in both models - using a convolution in the fundamental parameters model,

$$
f(\varepsilon)= \begin{cases}{[1 / \delta(2 \theta, \alpha)] \exp [\varepsilon / \delta(2 \theta, \alpha)]} & \varepsilon \leq 0 \\ 0 & \varepsilon>0\end{cases}
$$

and a $2 \theta$ offset equal to $-\delta(2 \theta, \alpha)$ in the TCHZ model, 


\section{short communications}

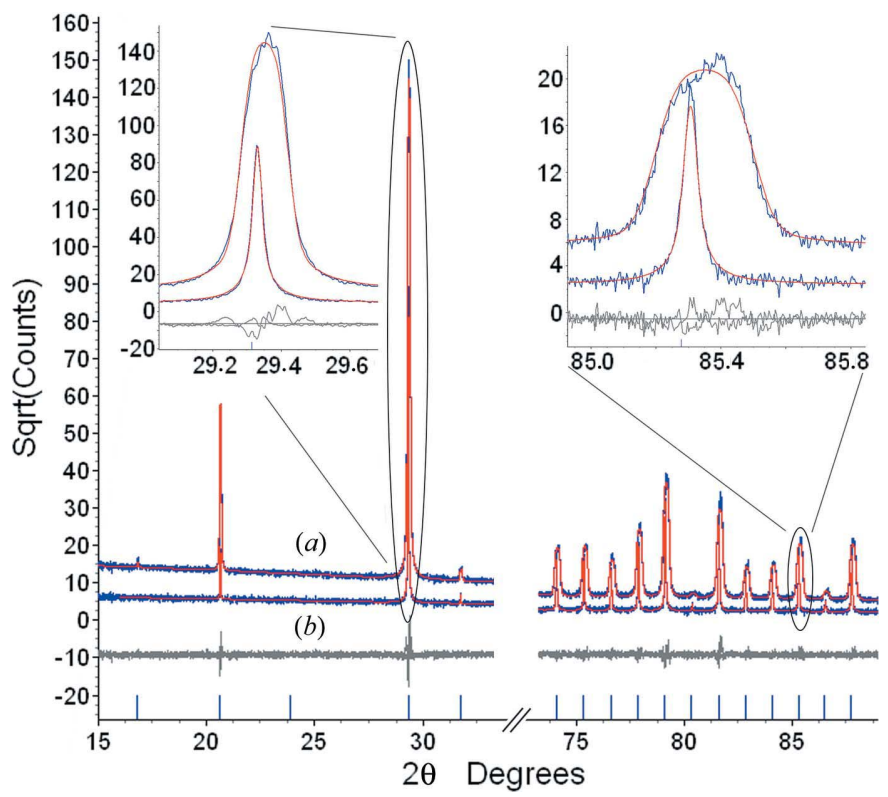

Figure 2

Fundamental parameters model. Experimental (blue) and calculated (red) diffraction patterns of yttrium oxide collected in the flat-plate asymmetric reflection geometry. Both patterns were fitted using the same instrumental and structural information. The beam heights and mixing parameters were refined. The peak offset is due to sample displacement. (a) Nominal beam height $=0.5 \mathrm{~mm}$, beam angle $=4 \cdot 0^{\circ}$. (b) Nominal beam height $=0.138 \mathrm{~mm}$, beam angle $=16^{\circ}$.

$$
\delta(2 \theta, \alpha)=\frac{1}{\mu R[\cot (2 \theta-\alpha)+\tan (\theta)]} \frac{180}{\pi} .
$$

In the fundamental parameters model, an independent parameter, $\eta$, constrained to be the same for those patterns collected with equal incident beam slit settings, was used to mix between purely hat and purely Gaussian incident beam profiles as described in equation (2). In the TCHZ model, the peak profile parameters $(U, V, W$ and $Z$ ) were constrained to be equal for all patterns.

The initial and refined parameters from both models are given in Table 1, as well as the $r_{\text {Bragg }}$ figures-of-merit for each of the patterns. These figures-of-merit show an average $12 \%$ improvement in the profile fit between the fundamental parameters approach and the empirical TCHZ model. Two indicative calculated patterns and the corresponding experimental data from both models are presented in Figs. 2 and 3. In the fundamental parameters model, the change in the mixing parameter shows how the distribution of intensity along the beam footprint varies with beam height. With a large beam height, the middle of the footprint is of uniform intensity, best approximated with a hat, with the fall off in intensity at the edges of the beam being a relatively small component, but having a Gaussian distribution. As the beam height decreases, the edges of the beam take up proportionally more of the footprint, altering the overall intensity profile towards a Gaussian.

\section{Conclusions}

It has been shown that a simple, physically based model, with up to three parameters, can improve the whole-pattern profile fitting of diffraction data collected in flat-plate asymmetric reflection geometry. The use of this model decreases the total number of parameters that are needed for accurate calculation of peak shape and width and, therefore, has the potential to improve refinement stability. The application of this beam-width correction, in conjunction with the appropriate sample displacement and

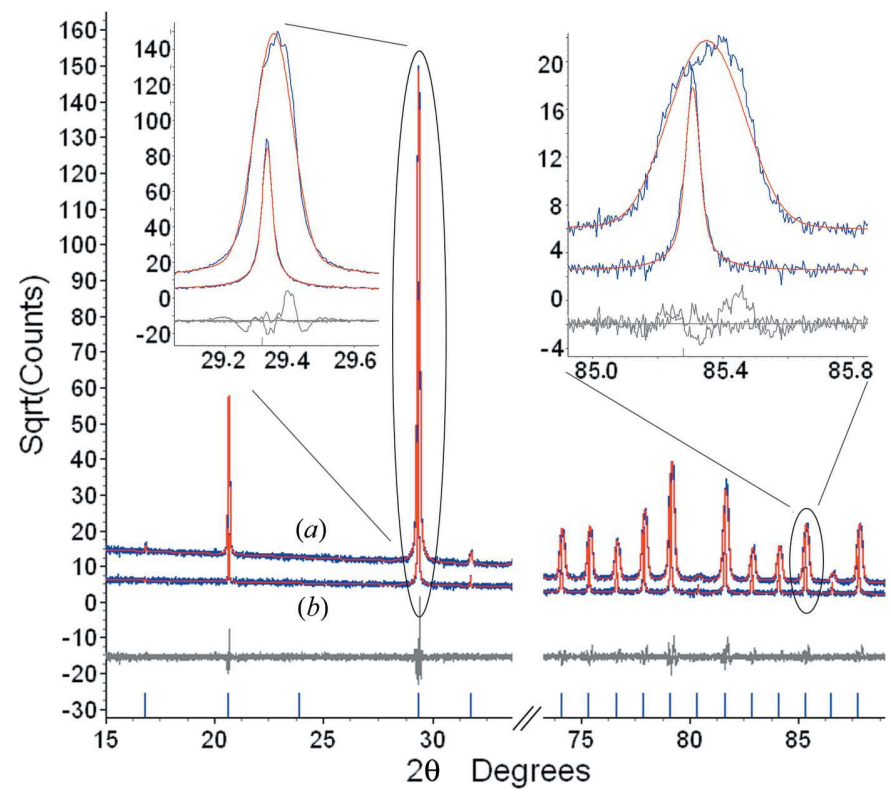

Figure 3

TCHZ model. Experimental (blue) and calculated (red) diffraction patterns of yttrium oxide collected in the flat-plate asymmetric reflection geometry. Both patterns were fitted using the same instrumental and structural information. The beam heights were refined. The peak offset is due to sample displacement. (a) Nominal beam height $=0.5 \mathrm{~mm}$, beam angle $=4.0^{\circ}$. (b) Nominal beam height $=$ $0.138 \mathrm{~mm}$, beam angle $=16^{\circ}$.

transparency (Masson et al., 1996) and intensity (Toraya et al., 1993) corrections, changes the inappropriate assumption of Bragg-Brentano or capillary geometry coded into the majority of Rietveld analysis programs.

Because of the strong correlation between beam height and beam angle, at least one of these parameters should be fixed to their measured values, rather than being refined as part of model development. Any additional peak broadening in the unknown samples should be due to crystallite size, strain or other such sample-dependent parameters. Additionally, if using the TCHZ model, the approach of Young \& Desai (1989) of separating peak profile parameters into instrument and sample components should be followed.

The authors wish to acknowledge the Australian Synchrotron (grant AS092/PD1329) for beamtime and powder diffraction beamline scientists Drs Justin Kimpton and Qinfen Gu for their assistance with data collection. This work was conducted under the auspices of the CSIRO Light Metals Flagship.

\section{References}

Alexander, L. E. (1954). J. Appl. Phys. 55, 155-161.

Bruker (2008). Diffracplus TOPAS. Bruker AXS, Karlsruhe, Germany.

Cheary, R. W. \& Coelho, A. (1992). J. Appl. Cryst. 25, 109-121.

Ishibashi, H., Shimomoto, K. \& Nakahigashi, K. (1994). J. Phys. Chem. Solids, $\mathbf{5 5}, 809-814$.

Masson, O., Guinebretière, R. \& Dauger, A. (1996). J. Appl. Cryst. 29, 540-546. Rietveld, H. M. (1969). J. Appl. Cryst. 2, 65-71.

Schmitt, B., Brönnimann, C., Eikenberry, E. F., Gozzo, F., Hörmann, C., Horisberger, R. \& Patterson, B. (2003). Nucl. Instrum. Methods Phys. Res. Sect. A, 501, 267-272.

Thompson, P., Cox, D. E. \& Hastings, J. B. (1987). J. Appl. Cryst. 20, 79-83. Toraya, H., Huang, T. C. \& Wu, Y. (1993). J. Appl. Cryst. 26, 774-777.

Toraya, H. \& Yoshino, J. (1994). J. Appl. Cryst. 27, 961-966.

Wallwork, K. S., Kennedy, B. J. \& Wang, D. (2007). Ninth International Conference on Synchrotron Radiation Instrumentation, edited by J.-Y. Choi \& S. Rah, pp. 879-882. Daegu: American Institute of Physics.

Young, R. A. \& Desai, P. (1989). Arch. Nauk. Mater. 10, 71-90. 